Ann. Sci. Forest., 1977, 34 (3), 205-214.

\title{
Contribution à la mesure des pertes de production causées par la chenille processionnaire (Thaumetopoea pityocampa Schiff.) au Pin maritime dans les Landes de Gascogne
}

\author{
B. LEMOINE \\ avec la collaboration d'A. SARTOLOU pour la partie technique \\ et $A$. BOULBRIA pour la partie entomologique \\ Laboratoire de Sylviculture et d'Ecologie de la Pinède landaise \\ Centre de Recherches de Bordeaux, I.N.R.A. \\ Domaine de l'Hermitage, 33610 Cestas
}

\begin{abstract}
Résumé
Cette étude, qui concerne une attaque isolée dans le temps, porte sur 773 arbres appartenant à 6 couples de lots de Pin maritime : lot complètement défeuillé par la chenille processionnaire d'une part, lot indemne d'autre part.

Les résultats sont les suivants :

a) Au plan de l'interprétation statistique on peut réduire considérablement l'erreur expérimentale résultant de la méthode par couples : il faut attendre que les croissances soient redevenues normales et alors procéder à un réajustement de la vigueur du lot attaqué par rapport à celle du lot témoin.

b) La période de récupération, au bout de laquelle les arbres recouvrent leur croissance normale, est de 3 ans.

c) La perte de croissance en volume, circonférence ef hauteur est de 31 p. 100 dans les cas les plus fréquents.

Ces deux résultats (b) et (c) sont conformes à ceux des autres auteurs.

d) on peut observer parfois des pertes de production supérieures mais pour des causes inconnues
\end{abstract}

\section{1. - Introduction}

Le but de cette étude est de préciser l'« anatomie» d'une attaque isolée dans le temps. Dans les Landes de Gascogne en effet on ne trouve pas en général de peuplement attaqué à quelques années d'intervalle, sauf en ce qui concerne certaines forêts du Marensin et de la région d'Arcachon. C'est en ce sens, et par la méthodologie particulière qui en découle, que notre étude diffère de celle de Bouchon J. et Toth J. (1971) dont l'objet d'étude est une pinède soumise à des gradations périodiques du ravageur.

Cette étude a été financée par la D. G. R. S. T. (Comité Lutte Biologique) Contrat n ${ }^{\circ} 70.02175$

Annales des Sciences forestières. - 1977 


\section{2. - Matériel et Méthode}

Afin de préciser à la fois l'intensité et la durée des effets nous avons mesuré tous les ans (à partir de la $2^{\mathrm{e}}$ année suivant l'attaque) les accroissements sur plusieurs couples de lots d'arbres:

- ayant subi une défeuillaison complète (note 4);

- étant restés indemnes d'attaque (note 0 ) ou ayant subi une attaque insignifiante (note 1) ;

Les 2 éléments du couple (lot complètement défeuillé et lot témoin) sont des lots, soit appartenant à 2 placeaux jugés les plus ressemblants possibles (âge, station, vigueur, sylviculture voire sylviculteur), soit constitués d'arbres en mélange pied à pied dans le même peuplement. Dans cette dernière situation le lot attaqué est toujours sensiblement plus vigoureux que le lot indemne ; c'est également le cas des arbres de la lisière attaquée comparés aux arbres témoins situés à l'intérieur du peuplement.

Chacun des lots comportant en fait non seulement les degrés extrêmes d'attaque (nulle ou totale) seuls étudiés ici, mais aussi tous les cas intermédiaires, une notation de 0 à 4 fut mise au point par l'entomologiste du Laboratoire ; sa signification au plan défeuillaison est la suivante :

\begin{tabular}{|c|c|}
\hline $\begin{array}{l}\text { Note } \\
\text { (Note) }\end{array}$ & $\begin{array}{l}\text { p. } 100 \text { défeuillaison } \\
\text { (en moyenne) } \\
\text { (p. } 100 \text { of defoliation) }\end{array}$ \\
\hline 0 & nul (nil) \\
\hline 1 & $\leqslant 5$ p. 100 \\
\hline 2 & 25 p. 100 \\
\hline 3 & 50 p. 100 \\
\hline 4 & \# 100 p. 100 \\
\hline & $\begin{array}{c}\text { (sauf aiguilles apicales } \\
\text { restées en place) } \\
\text { (excepted no eaten apex } \\
\text { needles) }\end{array}$ \\
\hline
\end{tabular}

Cette non-linéarité de la correspondance entre les nombres des 2 colonnes peut paraître paradoxale. Tout se passe comme si les degrés successifs appréciés variaient suivant la progression géométrique de raison 2. A posteriori nous pensons qu'il peut s'agir d'une manière réaliste de noter : les différences de dégâts variant du simple au double sont plus perceptibles que celles variant à un pas constant, par exemple de 25 p. 100.

Le Pin maritime laissant bien voir les aiguilles d'âges différents (de 1 à 3 ans) il fut possible d'utiliser rétrospectivement cette notation pour chaque couple de lots d'arbres mélangés pied à pied, là où bien entendu l'attaque avait été observée un an avant mais seulement qualitativement.

Ce système de notation mis au point lors de l'attaque principale a été utilisé pour juger de l'importance de l'attaque secondaire survenant 1 ou 2 ans après l'attaque principale. Cette importance étant relativement faible on attribue les effets à l'attaque 
TABLEAU 1

Caractéristiques des lots complètement défeuillés (1res lignes) et de leurs lots témoins (2e lignes)

Characteristics of lots completely defoliated (1st lines) and corresponding control plcts (2d lines)

\begin{tabular}{|c|c|c|c|c|c|c|c|c|c|c|c|}
\hline \multirow{2}{*}{$\begin{array}{l}\text { Placeau } \\
\text { (Plot) }\end{array}$} & \multirow{2}{*}{$\begin{array}{l}\text { Dispositif } \\
\text { (Design) }\end{array}$} & \multicolumn{2}{|c|}{$\begin{array}{c}\text { Attaque } \\
\text { principale } \\
\text { (Main } \\
\text { defoliation) }\end{array}$} & \multicolumn{2}{|c|}{$\begin{array}{l}\text { Attaque } \\
\text { secondaire } \\
\text { (Secondary } \\
\text { defoliation) }\end{array}$} & \multicolumn{2}{|c|}{$\begin{array}{l}\text { Eclaircie } \\
\text { ultérieure } \\
\text { (Thinnings) }\end{array}$} & \multirow{2}{*}{$\begin{array}{l}\text { Age initial } \\
\text { (Previous } \\
\text { pine age) }\end{array}$} & \multirow{2}{*}{$\begin{array}{c}\text { Nombre d'arbres } \\
\text { du lot } \\
\text { (Lot pines } \\
\text { number) }\end{array}$} & \multicolumn{2}{|c|}{$\begin{array}{l}\text { Dimension moyenne } \\
\quad \text { initiale } \\
\text { (Previous mean sizes) }\end{array}$} \\
\hline & & Année & Note & Année & Note & Année & $\begin{array}{l}\text { Prélèvement } \\
\text { p. } 100\end{array}$ & & & $\begin{array}{l}\text { Circonf. } \\
\text { (Circ.) } \\
\mathrm{cm}\end{array}$ & $\begin{array}{c}\text { Hauteur } \\
\text { (Height) } \\
\text { m }\end{array}$ \\
\hline $\begin{array}{l}\text { Parentis } 1 \\
\text { Parentis } 2\end{array}$ & Placeaux (Plots) & $\begin{array}{c}1969 \\
-\end{array}$ & $\begin{array}{l}4 \\
0\end{array}$ & $\begin{array}{r}1971 \\
1970-1971 \\
\end{array}$ & $\begin{array}{l}1,1 \\
0,4\end{array}$ & $\begin{array}{c}1970 \\
-\end{array}$ & $\stackrel{?}{-}$ & $\begin{array}{l}16 \\
16\end{array}$ & $\begin{array}{l}145 \\
109\end{array}$ & $\begin{array}{l}41 \\
40\end{array}$ & $\begin{array}{l}8,6 \\
8,5\end{array}$ \\
\hline $\begin{array}{l}\text { Parentis } 3 \\
\text { Parentis } 3\end{array}$ & $\begin{array}{l}\text { Pied à pied (Tree to } \\
\text { tree) }\end{array}$ & $\begin{array}{c}1969 \\
-\end{array}$ & $\begin{array}{l}4 \\
0\end{array}$ & $\begin{array}{l}1971 \\
1971\end{array}$ & $\begin{array}{l}0,5 \\
0,3\end{array}$ & - & - & $\begin{array}{l}20 \\
20\end{array}$ & $\begin{array}{l}54 \\
92\end{array}$ & $\begin{array}{l}36 \\
32\end{array}$ & $\begin{array}{l}7,4 \\
6,4\end{array}$ \\
\hline $\begin{array}{l}\text { Hume } 6 \\
\text { Hume } 5\end{array}$ & Placeaux (Plots) & $\begin{array}{c}1969 \\
-\end{array}$ & $\begin{array}{l}4 \\
0\end{array}$ & $\begin{array}{l}1970-1971 \\
1970-1971\end{array}$ & $\begin{array}{l}0,5 \\
0,1\end{array}$ & $\begin{array}{l}1971 \\
1971\end{array}$ & $\begin{array}{l}29 \\
35\end{array}$ & $\begin{array}{l}19 \\
19\end{array}$ & $\begin{array}{r}54 \\
104\end{array}$ & $\begin{array}{l}47 \\
54\end{array}$ & $\begin{array}{r}9,1 \\
10,7\end{array}$ \\
\hline $\begin{array}{l}\text { Léon } \\
\text { Léon }\end{array}$ & $\begin{array}{l}\text { Pied à pied (Tree to } \\
\text { tree) }\end{array}$ & $\begin{array}{c}1969 \\
-\end{array}$ & $\begin{array}{l}4 \\
0\end{array}$ & \begin{tabular}{|l|}
$1970-1971$ \\
$1970-1971$
\end{tabular} & $\begin{array}{l}0,3 \\
0,1\end{array}$ & $\begin{array}{l}1971 \\
1971\end{array}$ & $\begin{array}{l}7 \\
7\end{array}$ & $\begin{array}{l}46 \\
46\end{array}$ & $\begin{array}{l}13 \\
23\end{array}$ & $\begin{array}{l}99 \\
94\end{array}$ & - \\
\hline $\begin{array}{l}\text { Ligniculture } \\
\text { Ligniculture }\end{array}$ & Lignes (Rows) & $\begin{array}{c}1970 \\
-\end{array}$ & $\begin{array}{l}4 \\
0\end{array}$ & $\begin{array}{l}1971 \\
1971\end{array}$ & $\begin{array}{l}0,1 \\
0,1\end{array}$ & - & - & $\begin{array}{l}9 \\
9\end{array}$ & $\begin{array}{l}13 \\
20\end{array}$ & $\begin{array}{l}43 \\
42\end{array}$ & $\begin{array}{l}7,2 \\
6,4\end{array}$ \\
\hline $\begin{array}{l}\text { Lisière } \\
\text { Intérieur }\end{array}$ & Placeaux (Plots) & $\begin{array}{c}1970 \\
-\end{array}$ & $\begin{array}{l}4 \\
0\end{array}$ & & $\begin{array}{l}0,1 \\
0,5\end{array}$ & - & - & $\begin{array}{l}18 \\
18\end{array}$ & $\begin{array}{l}57 \\
49\end{array}$ & $\begin{array}{l}60 \\
49\end{array}$ & - \\
\hline
\end{tabular}


principale. On peut donc considérer avoir eu affaire à un phénomène isolé dans le temps.

Les paramètres de croissance mesurés sont la circonférence et la hauteur, la 1 re chaque fois et sur chaque placeau, la $2^{\mathrm{e}}$ au gré de nos possibilités en temps, mais suffisamment pour établir une bonne correspondance entre les effets sur la première et les effets sur la seconde.

Le tableau 1 récapitule les caractéristiques que nous venons de décrire.

\section{3. - Résultats}

Ils ne concernent que les lots d'arbres complètement défeuillés jugés par rapport à leurs témoins respectifs. Les cas d'arbres partiellement défeuillés ne se retrouvant pas en effet dans chaque parcelle du dispositif il est impossible de procéder à une analyse sérieuse de leurs pertes de croissance comme a pu le faire Cadahia et al. (1970) dans son expérience.

\section{1. - Perfes d'accroissement sur la circonférence}

Quand les éléments de chaque couple sont strictement comparables (à supposer qu'on puisse le vérifier) au point de vue lois et vigueur de croissance juste avant l'attaque la seule étude de l'évolution du rapport:

Accroissement annuel du lot défeuillé.

Accroissement annuel du lot témoin doit renseigner pleinement sur l'évolution du rapport $\frac{\text { Accroissement réel }}{\text { Accroissement potentiel }}$ du lot affecté par le ravageur. Cette évolution est appelée à chiffrer la durée et l'importance des dégâts.

On constate que ce rapport (cf. tabl. 2) se stabilise la 4e année après l'attaque autour d'une valeur moyenne qui n'est pas différente statistiquement de l'unité. II semble donc bien que la durée des dégâts soit, en moyenne, de 3 ans, période au bout de laquelle les arbres retrouvent leur croissance potentielle.

Cependant ce rapport $\tau_{4}$ ou $\tau_{5}$ affèrent à la $4 \mathrm{e}$ ou à la $5^{\mathrm{e}}$ année de même que les rapports $\tau_{3}$ et $\tau_{2}$ affèrenis à la $3^{\mathrm{e}}$ et à la $2^{\mathrm{e}}$ année, comporte une variabilité importante (du simple au double).

La méthode des couples présente donc une erreur expérimentale très forte qui confère à l'estimation des dégâts un intervalle de confiance trop lâche.

Si on considère l'attaque 1969 pour laquelle on a mis en œuvre 4 couples on voit que les variations statistiques de $\tau_{2}$ et de $\tau_{5}$ sont liées (cf. fig. 1) et que l'on peut envisager l'ajustement $\tau_{2} / \tau_{5}=\mathrm{k}$. En ce qui concerne l'attaque 1970, pour laquelle on n'a mis en œuvre que 2 couples, rien ne justifie le rejet d'un ajustement similaire (les résidus des 2 ajustements sont du même ordre de grandeur), d'où $\tau_{2} / \tau_{5}=k^{\prime}$. Cet ajustement permet de calculer pour chaque couple la valeur de $\tau_{2}$ si $\tau_{5}=1$ (cf. graphique 1) c'est-à-dire après avoir strictement rendu comparables les vigueurs instantanées du lot attaqué et du lot témoin ; cette démarche n'est pas différente dans son principe de celle de Bouchon J. et Toth J. (1971). 
TABLEAU 2

Accroissements annuels en circonférence IC des lots complètement défeuillés (1res lignes) et de leurs lots témoins (2es lignes). Rapport $\tau$ de ces 2 accroissements

Annual increments (circonference) lc of completely defoliated lots (1st lines) and of their control lots (2d lines). Ratio $\tau$ of this 2 increments

\begin{tabular}{|c|c|c|c|c|c|c|c|c|c|c|c|c|}
\hline \multirow{2}{*}{$\begin{array}{l}\text { Placeau } \\
\text { Plat }\end{array}$} & \multicolumn{3}{|c|}{$\begin{array}{c}\text { 2e année après } \\
\text { l'attaque } \\
\text { 2d year after attack }\end{array}$} & \multicolumn{3}{|c|}{$\begin{array}{c}\text { 3e année après } \\
\text { I'attaque } \\
\text { 3d year after attack }\end{array}$} & \multicolumn{3}{|c|}{$\begin{array}{c}\text { 4e année après } \\
\text { l'attaque } \\
\text { 4th year after attack }\end{array}$} & \multicolumn{3}{|c|}{$\begin{array}{c}5 \text { e année après } \\
\text { l'attaque } \\
\text { 5th year after attack }\end{array}$} \\
\hline & $\begin{array}{l}I_{2} C_{2} \\
\mathrm{~cm}\end{array}$ & $\tau_{2}$ & $\tau_{2}: \tau_{5}$ & $\begin{array}{l}\mathrm{IC}_{3} \\
\mathrm{~cm}\end{array}$ & $\tau_{3}$ & $\tau_{3}: \tau_{5}$ & $\underset{\mathrm{cm}}{\mathrm{IC}_{4}}$ & $\tau_{4}$ & $\tau_{4}: \tau_{5}$ & $\begin{array}{l}1 C_{5} \\
\mathrm{~cm}\end{array}$ & $\tau_{5}$ & $\tau_{5}: \tau_{5}$ \\
\hline $\begin{array}{l}\text { Parentis } 1 \\
\text { Parentis } 2\end{array}$ & $\begin{array}{l}1,48 \\
1,82\end{array}$ & 0,81 & 0,65 & $\begin{array}{l}2,38 \\
2,47\end{array}$ & 0,96 & 0,77 & $\begin{array}{l}2,49 \\
2,25\end{array}$ & 1,11 & 0,90 & $\begin{array}{l}2,66 \\
2,15\end{array}$ & 1,24 & 1,00 \\
\hline $\begin{array}{l}\text { Parentis } 3 \\
\text { Parentis } 3\end{array}$ & $\begin{array}{l}1,62 \\
2,38\end{array}$ & 0,68 & 0,71 & $\begin{array}{l}2,28 \\
2,44\end{array}$ & 0,94 & 0,98 & $\begin{array}{l}2,04 \\
2,12\end{array}$ & 0,96 & 1,00 & $\begin{array}{l}1,97 \\
2,06\end{array}$ & 0,96 & 1,00 \\
\hline $\begin{array}{l}\text { Hume } 6 \\
\text { Hume } 5\end{array}$ & $\begin{array}{l}0,87 \\
1,53\end{array}$ & 0,57 & 0,77 & $\begin{array}{l}2,01 \\
2,81\end{array}$ & 0,72 & 0,97 & $\begin{array}{l}2,46 \\
3,21\end{array}$ & 0,77 & 1,04 & $\begin{array}{l}3,20 \\
4,32\end{array}$ & 0,74 & 1,00 \\
\hline $\begin{array}{l}\text { Léon } \\
\text { Léon }\end{array}$ & $\begin{array}{l}0,29 \\
0,93\end{array}$ & 0,31 & 0,61 & $\begin{array}{l}0,57 \\
1,43\end{array}$ & 0,40 & 0,78 & $\begin{array}{l}1,11 \\
1,71\end{array}$ & 0,65 & 1,27 & $\begin{array}{l}0,47 \\
0,92\end{array}$ & 0,51 & 1,00 \\
\hline $\begin{array}{l}\text { Ligniculture } \\
\text { Ligniculture }\end{array}$ & $\begin{array}{l}0,45 \\
1,98\end{array}$ & 0,23 & 0,27 & $\begin{array}{l}2,04 \\
3,85\end{array}$ & 0,53 & 0,63 & $\begin{array}{l}3,67 \\
4,82\end{array}$ & 0,76 & 0,90 & $\begin{array}{l}2,18 \\
2,60\end{array}$ & 0,84 & 1,00 \\
\hline $\begin{array}{l}\text { Lisière } \\
\text { Intérieur }\end{array}$ & $\begin{array}{l}0,43 \\
2,30\end{array}$ & 0,18 & 0,15 & $\begin{array}{l}2,26 \\
3,73\end{array}$ & 0,61 & 0,50 & $\begin{array}{l}4,58 \\
4,21\end{array}$ & 1,09 & 0,90 & $\begin{array}{l}2,40 \\
1,99\end{array}$ & 1,21 & 1,00 \\
\hline
\end{tabular}

On constate alors que les moyennes $\overline{\mathrm{k}}$ pour l'attaque 69 et $\overline{\mathrm{k}^{\prime}}$ pour l'attaque 70 sont significativement différentes $(\mathrm{F} 1 / 4=55 * *)$. La perte de croissance $1-\overline{\mathrm{k}^{\prime}}$ due à l'attaque 70 serait donc plus forte que la perte $1-\bar{k}$ due à l'attaque 69 . Ce résultat important sera discuté plus loin.

\section{2. - Pertes d'accroissement sur la hauteur}

Nous proposons une excellente relation statistique entre les $\tau_{H}$ relatifs à la hauteur et les $\tau_{c}$ relatifs à la circonférence calculée pour les 2 attaques. On dispose en effet des mesures conjointes de ces 2 paramètres pour 4 couples et 4 années de mesures. On a la régression :

$$
\tau_{\mathbf{H}}=0,79 . \tau_{\mathbf{c}}+0,15 \text { avec } r=0,85 * * * .
$$

$\mathrm{Si}$ on considère par exemple la $2^{\mathrm{e}}$ année après l'attaque 69 faisant $\tau_{\mathrm{c}}=\overline{\mathrm{k}}=0,69$, on obtient à l'aide de cette relation $\overline{\mathrm{k}}=\tau_{\mathrm{H}}=0,70$ en sorte que l'on peut dire que les pertes de croissance en hauteur et en circonférence sont du même ordre de grandeur.

\section{3. - Pertes d'accroissement sur le volume}

On prend pour modèle de cubage de l'arbre moyen du lot la relation :

$$
V=\frac{f}{4 \pi} \cdot C^{2} \cdot H
$$




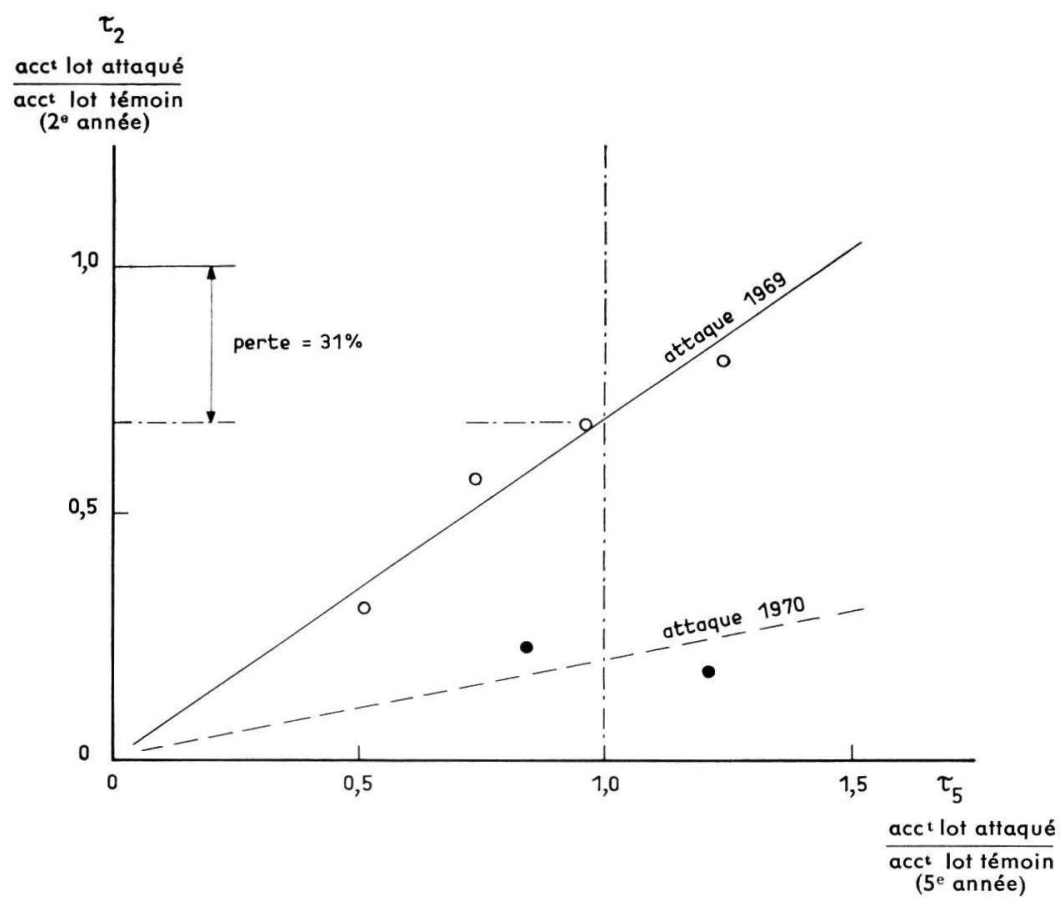

FIG. 1. - Réduction de l'erreur expérimentale du dispositif par couples.

Rapports $\frac{\operatorname{acc}{ }^{t} \text { lot attaqué }}{\operatorname{acc} \text { lot témoin }}$ : ajustement de la $2^{\mathrm{e}}$ année (période de récupération) sur la $5^{\mathrm{e}}$ année (après récupération).

Réduction of experimental error of couples design.

Ratios $\frac{\text { attacked incremt lot }}{\text { control incremt lot }}$ fit $2 d$ year (recovery period) on 5 th year (after recovery).

et admettant que $f$ ne subit pas de variations notables à l'échelle à laquelle nous travaillons, nous prenons comme modèle de cubage des accroissements courants du lot témoin la formule d'approximation suivante :

$$
\frac{\mathrm{IV}}{\mathrm{V}}=\frac{2 \mathrm{IC}}{\mathrm{C}}+\frac{\mathrm{IH}}{\mathrm{H}}
$$

et pour le lot attaqué :

$$
\rho \cdot \frac{\mathrm{IV}}{\mathrm{V}}=\tau\left(\frac{2 \mathrm{IC}}{\mathrm{C}}+\frac{\mathrm{IH}}{\mathrm{H}}\right) .
$$

On constate que le taux de réduction $\rho$ de l'accroissement courant en volume due à l'attaque est approximativement le même que le taux de réduction $\tau$ commun aux accroissements courants en circonférence et en hauteur. On vérifie par ailleurs que la formule d'approximation employée ne biaise ce résultat que de 1 à 2 p. 100. 
3.4. - Pertes d'accroissement pendant la durée des effets

La durée des effets est de 3 ans. Nous possédons bien les données de pertes d'accroissement pendant les $2^{\mathrm{e}}$ et $3^{\mathrm{e}}$ années suivant l'attaque, mais pas pendant la 1 re année. Cette étude devrait donc en toute rigueur être complétée par une analyse dendrochronologique. Cependant nous ne sommes ni les premiers ni les seuls à nous être penchés sur le problème.

Estimer la perte de la 1 re année revient alors à savoir de quel ordre de grandeur est celle-ci par rapport aux pertes respectives des $2^{2}$ et $3^{e}$ années. Joly (1970) et Cadahia et al. (1970) ont simulé une attaque isolée dans le temps, le 1re par défeuillaison artificielle, le second par infestation contrôlée. Dans les 2 expériences on observe le classement suivant:

$$
\text { perte } 1^{\text {re }} \text { année }>\text { perte } 2^{\mathrm{e}} \text { année }>\text { perte } 3^{\mathrm{e}} \text { année } .
$$

La $2^{\mathrm{e}}$ année, étant l'année médiane sur le plan des pertes, doit donc être une mesure assez bonne de l'ensemble des dégâts pendant la durée des effets. Ceci ne sera évidemment valable que si on a bien contrôlé que le processus d'attaque est conforme à la situation d'attaque isolée dans le temps. Selon les critiques qui nous ont été faites il se peut en effet qu'en cas d'attaque échelonnée sur 2 ans ce soit la perte de la 1 re année qui constitue la perte médiane.

Comme nous le verrons au paragraphe suivant, on est sûr que le processus d'attaque correspond bien à notre hypothèse seulement pour l'infestation 1969. Nous ne

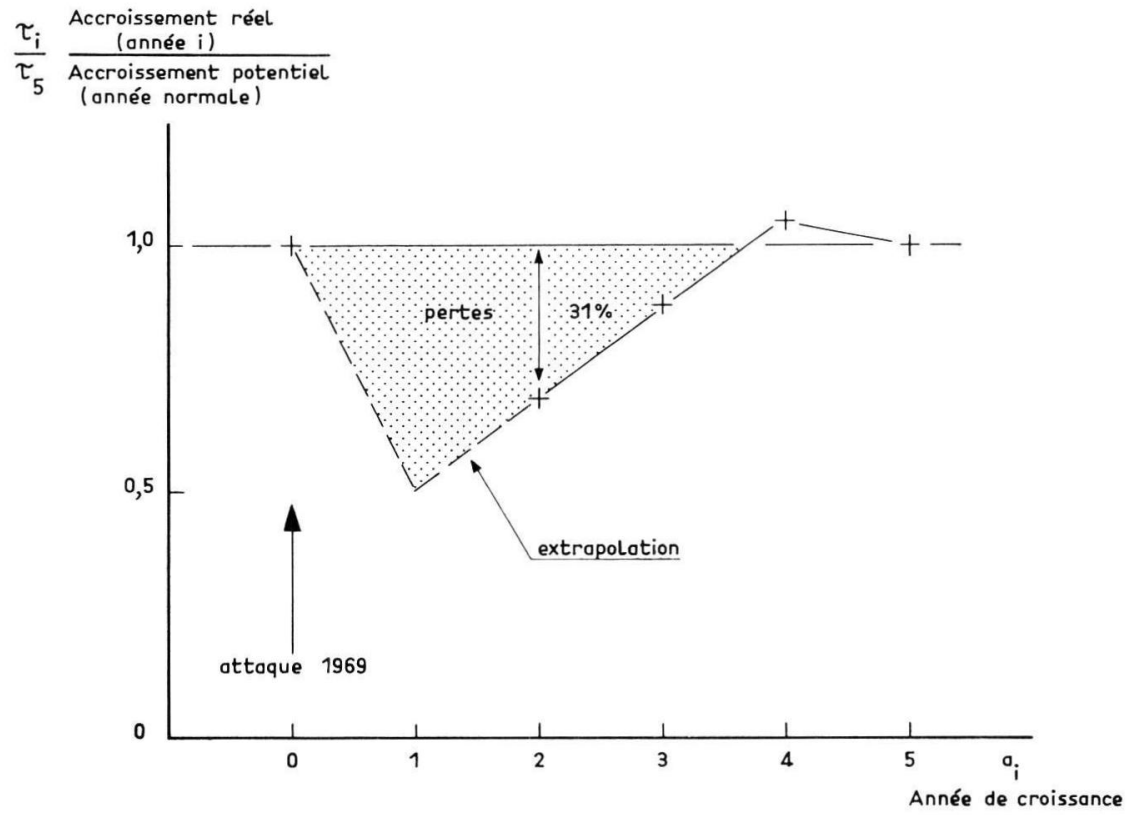

FIG. 2. - Estimation des pertes d'accroissement en circonférence pendant la durée des effets. Attaque 1969.

Estimated circonference increment losses during impact period. 1969 attack. 
faisons porter notre calcul que sur cette dernière. Admettre ce schéma simple c'est déterminer la perte pendant la 1 re année par l'extrapolation linéaire de la figure 2.

La perte d'accroissement en circonférence ainsi calculée est de 31 p. $100 \pm 11$ p. 100. Le chiffre de Bouchon J. et Toth J. (1971) est de 27 p. 100, qui tombe par conséquent dans notre intervalle de confiance. Notre perte d'accroissement en volume est aussi de 31 p. 100, or Cadahia et al. (1970) trouve 24 p. 100 sur des arbres plus jeunes devant également avoir récupéré leur croissance normale au bout de la 4 e année.

\section{4. - Discussion et conclusion}

La durée des effets, qualifiés par certains de période de récupération, est de 3 ans. On peut estimer que ceux de Cadahia et al. (1970) sont du même ordre de grandeur.

L'importance de l'effet s'est révélée plus forte à la suite de l'attaque 1970 qu'à la suite de l'attaque 1969. Deux hypothèses, non exclusives l'une de l'autre, sont avancées :

- mauvais contrôle de l'état sanitaire, antérieur à l'attaque étudiée, des lots ;

— interaction écologique des années.

La chenille processionnaire a pullulé dans les Landes en 1969 et 1970. Les placeaux sur lesquels on a étudié l'effet de l'attaque 1970 ont pu subir une $1^{\text {re }}$ attaque en 1969 (d'autant plus probable qu'il s'agit de peuplements de lisière ou presque), d'où la possibilité d'un effet cumulatif de 2 invasions même si on procède à un décalage de l'origine des temps pour le dispositif installé en 1970 (l'année 1 n'étant plus alors la saison de végétation 1970 mais celle de 1969).

Par ailleurs l'année climatologique 1970 révèle une sécheresse moyenne au printemps allant en s'accentuant jusqu'à l'automne, d'où une chute prématurée du cycle de croissance cambiale des arbres (cf. Lemoine B., 1975). Ces conditions écologiques ont pu avoir une répercussion plus grave sur des arbres au stade culminant de leur affaiblissement (venant de subir l'attaque) que sur des arbres en période de récupération. Cette sécheresse 1970 a donc pu agir plus fortement sur l'accroissement des premiers que sur l'accroissement des seconds non seulement immédiatement, mais encore une année plus tard par insuffisance des réserves constituées en fin de cycle.

La concordance de nos résultats concernant les effets de l'attaque 1969, qui a l'appui des hypothèses ci-dessus sont jugés bien plus représentatifs de la situation landaise normale que ceux de l'attaque 1970, avec ceux de Bouchon J. et Toth J., peut recevoir l'explication suivante. La pinède du Ventoux est soumise à des invasions périodiques séparées par des périodes de calme de 2 à 3 ans. Or on remarque que cette échelle de temps est précisément la même que celle de la période de récupération. Schématiquement les estimations des pertes d'accroissement effectuées sur le Pin noir auraient trait à une succession de 3 ans en 3 ans d'effets similaires à ceux que nous observons de façon isolée sur le Pin maritime. Il n'est donc pas étonnant que la perte relative de croissance observée pour toute la révolution du Pin noir soit la même que celle concernant à peine une rotation du Pin maritime.

Nos chiffres sont cohérents également avec ceux de Cadahia et al. (1970) qui résultent d'un processus d'invasion analogue au nôtre. 
Les résultats des divers auteurs, dont nous-même, convergent donc de façon très satisfaisante. C'est pour cela que, bien que notre intervalle de confiance soit assez lâche (10 p. 100), nous n'hésiterons pas à nous prononcer sur le chiffre de perte à retenir qui doit être 30 p. 100 ou 25 p. 100 pendant 3 ans.

Cette évaluation n'a trait qu'à l'arbre complètement défeuillé. L'estimation au niveau d'une parcelle est donc tributaire d'un inventaire. Le gestionnaire ne devra donc voir en ce travail qu'un élément de base devant l'aider dans ses décisions économiques.

Nous terminerons en soulignant que l'importance des dégâts peut être plus grande dans certains cas : les résultats de l'attaque 1970 sont là pour le rappeler. En sorte que si le calcul économique est fait à partir de notre résultat de 25 p. 100 ou 30 p. 100 il ne doit pas être notre seule préoccupation. L'interaction de l'année met peut-être en évidence un risque écologique important à voir attenter à la santé de la forêt par une combinaison de facteurs défavorables.

Reçu pour publication en février 1977.

\author{
Summary \\ Study of yield losses due to processionnary \\ caterpillar on maritime Pine in the Landes of Gascogne
}

This work dealing with a Thaumetopoea pityocampa single attack is based on 773 trees belonging to 6 couples of Pinus pinaster plots : one completely defoliated by the caterpillar, the another one not attacked.

The results are as follows :

a) For statistical interpretation the experimental error due to couple method can be reduced in a high degree : one must wait a renewal of normal increment and then compare the attacked plot vigor to control plot vigor.

b) The recovery lasts for 3 years before a new normal growth.

c) The loss of volume, circonference, and height increment is about 31 p. 100 in most cases.

These two results $(b)$ and $(c)$ are similar to those of others authors.

d) Higher losses can sometimes be observed but their causes are not known.

\title{
Références bibliographiques
}

BOUCHON J., TOTH J., 1971. Etude préliminaire sur les pertes de production des pinèdes soumises aux attaques de la processionnaire du pin Thaumetopoea pityocampa. Schiff. Ann. Sci. Forest., 1971,28 (3), 323-340.

CADAHIA D., INSUA A., 1970. Estimacion de los daños producidos por Thaumetopoea pityocampa Schiff. en repoblaciones de Pinus radiata D. Don. Bol. Del Serv. de Plagas Forestales, Año XIII, no 26, Madrid, 1970, 159-171.

JOLY R., 1970. Action des déprédations dues aux insectes defoliateurs sur le Pin maritime. Rev. Forest. Franç., XXII, no spécial, 1970, 205-209.

LEMOINE B., 1975. Essai de systhèse biomathématique des aspects concurrentiels, écologiques, morphologiques et cycliques de la croissance du Pin maritime dans la Pinède des Landes de Gascogne. Oecol. plant., 10 (2), 141-167. 